\title{
Palaeophytochemical Constituents from the Miocene-fossil Wood of Picea likiangensis in Xun-dian of Yunnan, China
}

\author{
You-Xing Zhao, Jian-Rong Luo, ${ }^{\dagger \neq}$ Cheng-Sen Li, ${ }^{\S}$ Tie-Mei Yi, and Jun Zhou, ${ }^{\dagger+*}$ \\ State Kev Laboratory of Phutochemistry and Plant Resources in West China, Kuming Institute of Botaty, \\ Chinese Academy of Sciences, Kumming 650204, China. "E-mail: jzhouamail kib.ac.on \\ ${ }^{\ddagger}$ Deparment of Pharmacology, Dali Universitw, Dali 671000, China \\ sinstitute of Botany, Chinese Academy of Sciences, Beijing 100093, China \\ Received Mav 2, 2008
}

Key Words : Miocene-fossil. Picea likiangensis. Constituents. 5-Phemyl-5,10.10a, I1-tetrahydro-4bH-benzo[b]fluorene-3,7-diol

The analysis of chemical constituents in specific plant fossils provides crucial information for their probable diagenetic pathways. Natural products in plant fossils could retain their characteristic basic structural skeletons and be used as chemosystematic marker or biomarkers for their biological origin. though they may undergo rapid diagenetic processes during their fossilization. " noids and other constituents were detected from Jurassic. Cretaceous, Pliocene, Miocene and Eocene plants, previously. ${ }^{3-8}$ Picea likiangensis is an economically important conifer indigenous to the southwest and center regions of China. ${ }^{9} \mathrm{~A}$ piece of Miocene-fossil wood of P. likiangensis. which was preserved morphologically, was discovered in an open Jinsuo coalnine in Xundian of Yunnan Province. China. raising questions about its phytochenical constituents. In this paper, we report the palaeophy tochenical investigation on the Miocene-fossil wood of $P$. likiangensis by phytochemical methods as column cliromatography to probe their organic constituents.
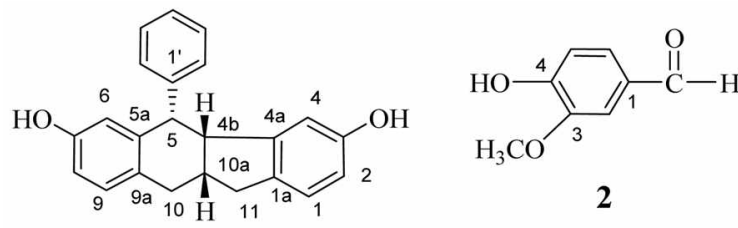

1
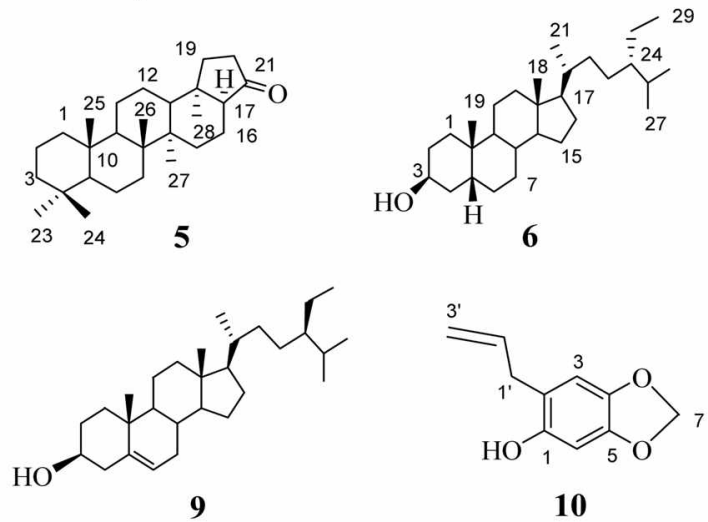

10

An ethanol extract prepared from the Miocene-fossil wood of $P$. likiangensis was purified repeatedly by column chromatography on silica gel and Sephadex LH-20 and then subjected to preparative thin-layer chromatography, yielding a new fluorene derivative (1) and other eleven known compounds (2-12) as shown in Figure 1 .

Compound 1 was obtained as amorphous powder, with a melting point (mp) of $121-123^{\circ} \mathrm{C}$. and its molecular formula $\mathrm{C}_{23} \mathrm{H}_{20} \mathrm{O}_{2}$ with fourteen unsaturation degrees was determined from a quasi-molecular ion peak at $m>328 \mathrm{in}$ its EI mass spectrum and the ${ }^{13} \mathrm{C}$-NMR (DEPT) spectrum. which was supported by its HR-ESI MS observed at $m z 351.1357$ (calculated $351.1360 .[\mathrm{M}+\mathrm{Na}]^{-}$). The IR spectrum of compound 1 showed the presence of aromatic rings (1611. 1494 and $1452 \mathrm{~cm}^{-1}$ ) and hydrosyl groups (3385 and 3477 $\mathrm{cm}^{-1}$ ). The ${ }^{1} \mathrm{H}-\mathrm{NMR}$ spectrum of compound 1 showed eleven aromatic protons among which six protons at $\delta 6.00$ (lH. d. $J=1.6 \mathrm{~Hz}), 6.47(\mathrm{lH}$. dd. $J=1.6,7.6 \mathrm{~Hz}) .6 .96(\mathrm{lH}$, d. $J=7.6 \mathrm{~Hz}), 6.15(\mathrm{lH}, \mathrm{d} . J=2.4 \mathrm{~Hz}) .6 .52(\mathrm{lH} . \mathrm{dd}, J=2.4$,

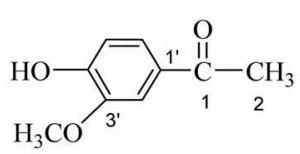

3
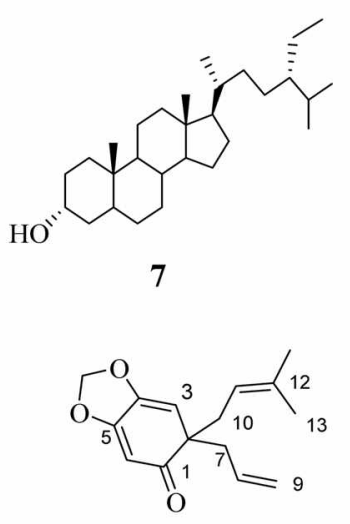

11

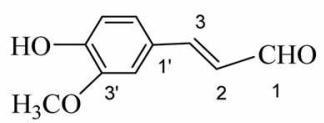

4
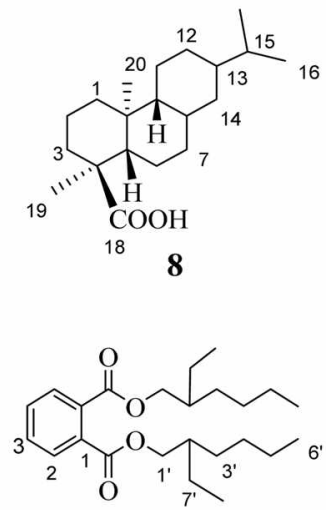

12

Figure 1. Structures of compounds 1-12 
$8.0 \mathrm{~Hz})$ and $6.94(1 \mathrm{H} . \mathrm{d}, J=8.0 \mathrm{~Hz})$ indicated two typical tri-substituted aromatic groups. The ${ }^{13} \mathrm{C}$-NMR and DEPT spectra of compound 1 revealed the presence of twenty-three carbon atoms including three aromatic rings and five saturated carbons. Two aromatic rings along with two methy lene groups at $\delta 36.1$ (t. C.10) and 37.6 (t. C.11) and three methine carbons at $\delta 54.4$ (d. C- 4 b). 53.4 (d. C.5) and 47.7 (d. C.10a) suggested the skeleton of tetrahydrobenzo[b] fluorine in 1, which was supported by the correlations of $\mathrm{H}-5$ $(\delta 3.98)$ with $\mathrm{C}-6(\delta 117.5)$, and $\mathrm{C}-10 \mathrm{a}(\delta 47.7): \mathrm{H}-10(\delta$ $2.98)$ with $\mathrm{C}-9(\delta 131.1), \mathrm{C}-5 \mathrm{a}(\delta 1+3.4), \mathrm{C}-1 \mathrm{l}(\delta 37.6)$ and $\mathrm{C}-4 \mathrm{~b}(\delta 54.4)$ : H-ll $(\delta 2.50$ and $2.9 \mathrm{l})$ with $\mathrm{C}-\mathrm{l}(\delta 125.5)$. C. $4 \mathrm{~b}(\delta 54.4) . \mathrm{C}-4 \mathrm{a}(\delta 148.2)$ and $\mathrm{C}-10(\delta 36.1)$; and $\mathrm{H}-4(\delta$ $6.00)$ with $\mathrm{C}-4 \mathrm{~b}(\delta 54.4)$ in the HMBC spectrum as shown in Figure 2 and the cross-peaks between $\mathrm{H}-10 / \mathrm{H}-10 \mathrm{a}, \mathrm{H}-\mathrm{Il} / \mathrm{H}$ 10a. $\mathrm{H}-10 \mathrm{a} / \mathrm{H}-4 \mathrm{~b}$. $\mathrm{H}-4 \mathrm{~b} / \mathrm{H}-5 . \mathrm{H}-1 / \mathrm{H}-2$ and $\mathrm{H}-8 / \mathrm{H}-9$ in the ${ }^{1} \mathrm{H}-{ }^{1} \mathrm{H}$ COSY spectrum. Another aromatic ring was attached to $\mathrm{C}-5$ based on the key HMBC correlations of $\mathrm{H}-5(\delta 3.98)$ with $\mathrm{C}-2$ and $6(\delta 130.8)$. The two hydroxyl groups were adjacent to $\mathrm{C}-3$ and $\mathrm{C}-7$ as established by analysis of coupling constants of $\mathrm{H}-4(\delta 6.00$, d. $J=1.6 \mathrm{~Hz})$ and $\mathrm{H}-6(\delta$ $6.15, \mathrm{~d}, J=2.4 \mathrm{~Hz}$ ). respectively. and the corresponding carbon chemical shifts. The relative stereochemistry of compound 1 was deduced by the ROESY spectrum exhibiting cross-peaks of $\mathrm{H}-5 / \mathrm{H}-10 \mathrm{a}, \mathrm{H}-5 / \mathrm{H}-4 \mathrm{~b}, \mathrm{H}-4 \mathrm{~b} / \mathrm{H}-10 \mathrm{a}, \mathrm{H}-5 / \mathrm{H}-6$. $\mathrm{H}-5 / \mathrm{H}-4$ and $\mathrm{H}-5 / \mathrm{H}-2$asshowninFigure 2 and Table 1. indicating the cis-orientations of $\mathrm{H}-5 / \mathrm{H}-4 \mathrm{~b}$ and $\mathrm{H}-4 \mathrm{~b} / \mathrm{H}-10 \mathrm{a}$ in 1. Based on the above evidence, the structure of compound 1 was identified as 5-phenyl-5.10.10a.11-tetrahydro-

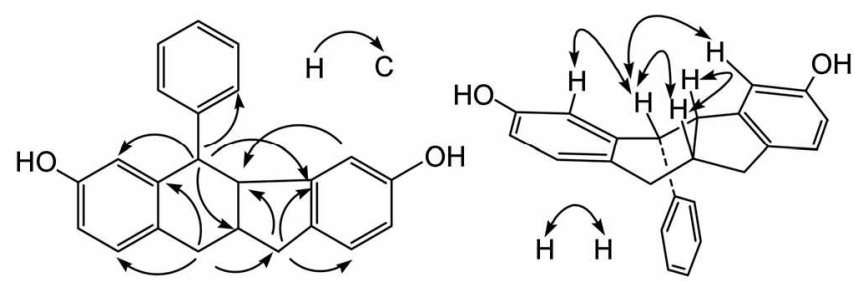

Figure 2. Selected HMBC and ROESY correlations of 1.

\section{4bH-benzo[b]fluorene-3,7-diol.}

Eleven known compounds (2-12) were elucidated to be 4hydroxy -3-methoxy benzaldehyde (2). ${ }^{11}$ acetovanillone (3) ${ }^{11}$ (Z)-3-(4-hydroxy-3-methoxyphenyl) acrylaldehyde (t). ${ }^{13}$ $22,29.30$-trinor-21-hopanone $(5)^{13} \quad 5 \beta$-24S-etlyylchlestan$3 \beta$-ol $(6){ }^{1+} 24 \alpha$-ethylchlestan- $3 \alpha$-ol $(7),{ }^{15}$ abietan- $18 \beta$-oic acid (8). ${ }^{16} 3 \beta$-sitosterol (9). ${ }^{17} 2$-allyl-4,5-(methylenedioxy) phenol (10). ${ }^{18}$ 6-allyl-6-(3-methylbut-2-enyl)benzo[d] [1.3] dioxol- $5(6 \mathrm{H})$-one $(11){ }^{19}$ bis (2-ethylhexyl)phthalate $(\mathbf{1 2})^{3 \mathrm{in}}$ according to the analysis of their spectral data and literature. respectively. Bis(2-etlyylhexyl)plthalate, a well-known plasticizer (DOP), belongs to phthalate diesters which are widely distributed in environment including animal tissues. excreta, food, and plastic containers. ${ }^{21.23}$ Phthalate diesters can easily migrate among media ${ }^{24}$ Compound 12 isolated from this Miocene-fossil wood of $P$. likiangensis here might be its inuer natural product or contaminated ones derived from enviromment such as coals and sediments during the storage and fossilization process in brown coal.

Table 1. ${ }^{1} \mathrm{H}^{13} \mathrm{C}$ NMR data and HMBC, ${ }^{1} \mathrm{H}-{ }^{1} \mathrm{H}$ COSY, ROESY conelations of 1 (400 MHz, in $\mathrm{CD}_{3} \mathrm{OD}$, $\delta$ ppm)

\begin{tabular}{|c|c|c|c|c|c|}
\hline No. & $\delta_{H}$ & $\delta$ & HMBC & $\mathrm{H}-\mathrm{H} \mathrm{COSY}$ & ROESY \\
\hline 1 & $6.96(\mathrm{~d}, 7.6 \mathrm{~Hz})$ & $125.5(\mathrm{~d})$ & $\mathrm{C}-2,3,4 \mathrm{a}, 11$ & $\mathrm{H}-2$ & $\mathrm{H}-2,11$ \\
\hline 2 & $6.47(\mathrm{dd}, 1.6,7.6 \mathrm{~Hz})$ & $114.0(\mathrm{~d})$ & $\mathrm{C}-3,4, \mathrm{Ia}$ & $\mathrm{H}-\mathrm{l}$ & $\mathrm{H}-\mathrm{l}$ \\
\hline 3 & - & $156.4(s)$ & - & - & - \\
\hline 4 & $6.00(\mathrm{~d}, 1.6 \mathrm{~Hz})$ & $112.4(\mathrm{~d})$ & $\mathrm{C}-2,3,1 \mathrm{a}, 4 \mathrm{~b}$ & - & $\mathrm{H}-5,4 \mathrm{~b}, 2^{\prime}, 6^{\prime}$ \\
\hline 5 & $3.98(\mathrm{~d}, 11.5 \mathrm{~Hz})$ & $53.4(\mathrm{~d})$ & $\mathrm{C}-6,5 \mathrm{a}, 4 \mathrm{~b}, 10 \mathrm{a}, \mathrm{I}^{\prime}, 2 ;, 6,4 \mathrm{a}, 9 \mathrm{a}$ & $\mathrm{H}-4 \mathrm{~b}$ & $\mathrm{H}-6,4 \mathrm{~b}, 10 \mathrm{a}, 2^{\prime}, 6^{\prime}$ \\
\hline 6 & $6.15(\mathrm{~d}, 2.4 \mathrm{~Hz})$ & $117.5(\mathrm{~d})$ & $\mathrm{C}-5,7,8,9 \mathrm{a}$ & - & $\mathrm{H}-5,2^{\prime}, 6^{\prime}$ \\
\hline 7 & - & $156.2(s)$ & - & - & - \\
\hline 8 & $6.52(\mathrm{dd}, 2.4,8.0 \mathrm{~Hz})$ & $114.4(d)$ & $\mathrm{C}-6,7,9 \mathrm{a}$ & $\mathrm{H}-9$ & $\mathrm{H}-9,10$ \\
\hline 9 & $6.94(\mathrm{~d}, 8.0 \mathrm{~Hz})$ & $131.1(\mathrm{~d})$ & $\mathrm{C}-7,10,5 \mathrm{a}$ & H-8 & $\mathrm{H}-8,10$ \\
\hline 10 & $2.98(\mathrm{~m})$ & $36.1(t)$ & $C-9,11,10 a, 4 b, 9 a, 5 a$ & $\mathrm{H}-10 \mathrm{a}$ & $\mathrm{H}-8,9,4 \mathrm{~b}, 10 \mathrm{a}, 11$ \\
\hline 11 & $\begin{array}{l}2.50(\mathrm{dd}, 11.4,13.9 \mathrm{~Hz}) \\
2.91(\mathrm{dd}, 6.9,13.9 \mathrm{~Hz})\end{array}$ & $37.6(t)$ & $\mathrm{C}-1,10,1 \mathrm{a}, 4 \mathrm{a}, 4 \mathrm{~b}, 10 \mathrm{a}$ & $\mathrm{H}-10 \mathrm{a}$ & $\mathrm{H}-1,10 \mathrm{a}, 10$ \\
\hline$l^{\prime}$ & - & $147.0(s)$ & - & - & - \\
\hline $2^{\prime}$ & $7.31(\mathrm{~d}, 7.5 \mathrm{~Hz})$ & $130.8(d)$ & $C-5,1^{\prime}, 3^{\prime}, 4^{\prime}, 6^{\prime}$ & $\mathrm{H}-3^{\prime}$ & $\mathrm{H}-4,5,6,4 \mathrm{~b}, 3^{\prime}, 5^{\prime}$ \\
\hline $3^{\prime}$ & $7.37(\mathrm{dd}, 7.4,7.5 \mathrm{~Hz})$ & $129.8(\mathrm{~d})$ & $C-1^{\prime}, 2^{\prime}, 4^{\prime}, 5^{\prime}$ & $\mathrm{H}-2,4$ & $\mathrm{H}-2^{\prime}, 4^{\prime}, 6^{\prime}$ \\
\hline 4 & $7.29(\mathrm{t}, 7.4 \mathrm{~Hz})$ & $127.6(\mathrm{~d})$ & $\mathrm{C}-2^{\prime}, 3^{\prime}, 5^{\prime}, 6^{\prime}$ & $\mathrm{H}-3^{\prime}, 5^{\prime}$ & $H-3^{\prime}, 5^{\prime}$ \\
\hline $5^{\prime}$ & $7.37(\mathrm{dd}, 7.4,7.5 \mathrm{~Hz})$ & $129.8(\mathrm{~d})$ & $C-1^{\prime}, 2^{\prime}, 4^{\prime}, 5^{\prime}$ & $\mathrm{H}-4^{\prime}, 6^{\prime}$ & $\mathrm{H}-2^{\prime}, 4^{\prime}, 6^{\prime}$ \\
\hline $6^{\prime}$ & $7.31(\mathrm{~d}, 7.5 \mathrm{~Hz})$ & $130.8(d)$ & $C-5,1^{\prime}, 3^{\prime}, 4^{\prime}, 6^{\prime}$ & H-5' & $\mathrm{H}-4,5,6,4 \mathrm{~b}, 3^{\prime}, 5^{\prime}$ \\
\hline la & - & $136.0(s)$ & - & - & - \\
\hline $4 a$ & - & $148.2(s)$ & - & - & - \\
\hline $4 b$ & $3.14(t, 11.5)$ & $54.4(d)$ & $C-4,5,10,1 \mathrm{a}, 4 \mathrm{a}, 5 \mathrm{a}, 10 \mathrm{a}, \mathrm{l}^{\prime}$ & $\mathrm{H}-5,10 \mathrm{a}$ & $\mathrm{H}-4,5,10,10 \mathrm{a}, 2^{\prime}, 6^{\prime}$ \\
\hline $5 \mathrm{a}$ & - & $143.4(s)$ & - & - & - \\
\hline $9 \mathrm{a}$ & - & $129.3(s)$ & - & - & - \\
\hline $10 \mathrm{a}$ & $2.22(\mathrm{~m})$ & $47.7(\mathrm{~d})$ & $\mathrm{C}-5,11,9 \mathrm{a}$, & $\mathrm{H}-10,11,4 \mathrm{~b}$ & $\mathrm{H}-5,10,11,4 \mathrm{~b}$ \\
\hline
\end{tabular}




\section{Experimental Section}

General experimental procedures. Melting points were measured on an XRC-1 micro-melting apparatus and are uncorrected. IR spectra were measured on a Bio-Rad FTS135 spectroneter with $\mathrm{KBr}$ pellets. UV spectra were recorded on a UV $210 \mathrm{~A}$ spectrometer. MS spectra were carried out on a VG Auto Spec-3000 spectrometer. The ID and 2D NMR spectra were run on Bruker AM-400 MHz and DRX$500 \mathrm{MHz}$ spectrometer using TMS as an intental. Silica gel (200-300 mesh, Marine Chemical Factory, Qingdao. China) were used for columin chromatography.

Plant materials. The Miocene-fossil wood of Picea likiangensis was collected from an open Jinsuo coalnine in Xundian of Yunnan Province. People's Republic of China. The identity of this fossil wood material was verified by professor Cheng-Sen Li and a voucher specinien (KMJS-01) has been deposited in the State Key Laboratory of Phytochemistry and Plant Resources in West China. Kumming Institute of Botany. Chinese Acadenty of Sciences.

Extraction and isolation. The Miocene-fossil wood powder of $P$. likiangensis $(23 \mathrm{Kg}$ ) was extracted with hot ethanol $(80 \mathrm{~L} \times 3)$ and filtered. The ethanol extraction was evaporated in vacuum to give the residue $(380 \mathrm{~g})$. The residue $(200 \mathrm{~g})$ was subjected to column cluromatography on silica gel (200-300 mesh), eluted with gradient petroleun etheracetone $(3: 2)$ to yield 5 fractions. Fraction $3(8 \mathrm{~g})$ was purified by repeated column cluromatography on silica gel with petroleun ether:acetone $(4: 1)$ and petroleum ether: EtOAc (5:1) and on Sephadex LH-20 with MeOH to afford conlpound 1 (5l mg). 2 (25 mg), $3(20 \mathrm{mg}$ ) and $+(22 \mathrm{mg})$. Fraction $1(3 \mathrm{lg})$ was separated by repeated column chromatography on silica gel with petroleun etheracetone (10:1) and petroleum ether: $\mathrm{CHCl}_{3}(\mathrm{I}: \mathrm{l})$ and on Sephadex LH-20 with $\mathrm{MeOH}: \mathrm{CHCl}_{3}(1: \mathrm{l})$ to give compound $5(6 \mathrm{mg}), 6(20 \mathrm{mg})$. 7 (25 $\mathrm{mg}) .8$ (12 $\mathrm{mg}$ ) and 9 (1.2 g). Fraction $2(4 \mathrm{~g})$ was subjected to columu chromatography on silica gel with petroleum ether:acetone $(8: 1)$ and petroleum ether: $\mathrm{CHCl}_{3}$ (1:2) and on Sephadex $\mathrm{LH}-20$ with $\mathrm{MeOH}: \mathrm{CHCl}_{3}$ (1:1) to yield compound 10 (15 mg). 11 ( $8 \mathrm{mg}$ ) and 12 (19 $\mathrm{mg})$.

5-Phenyl-5,10,10a,11-tetrahydro-4bH-benzo[b]fluorene3,7-diol (1). Amorphous powder. mp: $121-123^{\circ} \mathrm{C} ;[\alpha]_{D}^{3-4}$ +6.98 (c 0.21. $\mathrm{MeOH}$ ); IR (KBr) v3477. 3385. 1611. 1494 . $1452.1340,954,872.817,736,702 \mathrm{~cm}^{-1}$. UV $(\mathrm{MeOH}) \lambda_{\text {max }}$ $(\log \varepsilon): 204$ (3.19). 283 (2.24). 322 (0.99). 353 (1.01). 368 (0.79) nm; ${ }^{1} \mathrm{H}-\mathrm{NMR}\left(400 \mathrm{MHz} . \mathrm{CD}_{3} \mathrm{OD}\right)$ and ${ }^{13} \mathrm{C}-\mathrm{NMR}$ (100 MHz. $\mathrm{CD}_{j} \mathrm{OD}$ ) data see Table l: HRESIMS $m z$ : 351.1357 (calcd. for $\mathrm{C}_{23} \mathrm{H}_{2 j} \mathrm{O}_{2} \mathrm{Na}$. 351.1360): EIMS $m z$ (\%): $328[\mathrm{M}]^{+}(62) .251$ (4). $250(12), 221$ (32), 207 (19). $197(68) .196(90) .195(100), 179(36) .165(31) .107(27)$. 91 (19).

4-Hydroxy-3-methoxybenzaldehyde (2). Yellow needles $(\mathrm{MeOH}) .{ }^{1} \mathrm{H}-\mathrm{NMR}\left(500 \mathrm{MHz}, \mathrm{CDCl}_{j}\right) \delta 3.95$ (3H. s. $\left.\mathrm{OCH}_{3}\right) .7 .03$ (1H. dd. $\left.J=1.2 .8 .4 \mathrm{~Hz}, \mathrm{H}-6\right) .7 .79$ (1H. d. $J=$ $8.4 \mathrm{~Hz}, \mathrm{H}-5$ ). 7.43 (lH. d, $J=1.2 \mathrm{~Hz}, \mathrm{H}-2), 9.82$ (1H, s. CHO): EIMS $m z(\%): 152\left(\left[\mathrm{M}^{-}, 93\right), 149(40), 123(20)\right.$. $109(17) .93(14), 81(28), 69(41)$.
Acetovanillone (3). Yellow needles (MeOH). ${ }^{1} \mathrm{H}-\mathrm{NMR}$ $\left(400 \mathrm{MHz} . \mathrm{CDCl}_{3}\right): \delta 2.93(3 \mathrm{H}, \mathrm{s} . \mathrm{H}-2) .7 .32(\mathrm{lH}, \mathrm{d}, J=8.6$ Hz. H-5'), 7.90 (lH, d. $J=2.1 \mathrm{~Hz} . \mathrm{H}-2$ '). $7.9 \mathrm{l}(\mathrm{lH}, \mathrm{dd}, J=$ $\left.2.1,8.6 \mathrm{~Hz}, \mathrm{H}-6^{\prime}\right) .4 .31\left(3 \mathrm{H}, \mathrm{s} . \mathrm{OCH}_{3}\right)$ : EIMS $m z(\%): 166$ $\left([\mathrm{M}]^{+}, 49\right) .151(100), 123(26) .85(18)$.

(Z)-3-(4-Hydroxy-3-methoxyphenyl)acrylaldehyde (4). Yellow needles $(\mathrm{MeOH}) .{ }^{1} \mathrm{H}-\mathrm{NMR}\left(500 \mathrm{MHz} . \mathrm{CD}_{3} \mathrm{OD}\right) \delta$ $3.91\left(3 \mathrm{H} . \mathrm{s} . \mathrm{OCH}_{3}\right) .7 .24\left(\mathrm{lH} . \mathrm{d}, J=1.8 \mathrm{~Hz}, \mathrm{H}-2^{\prime}\right), 7.16(\mathrm{lH}$. dd, $\left.J=8.2,1.8 \mathrm{~Hz} . \mathrm{H}-6^{\prime}\right), 6.84$ (lH. d, $J=8.2 \mathrm{~Hz}, \mathrm{H}-5$ ), 9.56 (lH. d. $J=7.9 \mathrm{~Hz}, \mathrm{H}-1$ ), 7.57 (lH. d. $J=15.7 \mathrm{~Hz}, \mathrm{H}-3$ ), 6.64 (lH. dd. $J=15.7 .7 .9 \mathrm{~Hz}$. H-2): EIMS $m z(\%): 178$ ([M] ${ }^{-}$, 100), 177 (29). 163 (11). 161 (20). 135 (25). 107 (18).

22,29,30-Trinol-21-hopanone (5). White needles $(\mathrm{MeOH})$. ${ }^{1} \mathrm{H}-\mathrm{NMR}\left(500 \mathrm{MHz} . \mathrm{CDCl}_{3}\right) \delta 2.22(2 \mathrm{H}, \mathrm{t} . \mathrm{H}-20) .1 .80(1 \mathrm{H}$, t. H-17), 1.31 (3H, s. H-28). 1.18 (3H. s, H-27). 0.99 (3H, s, H-26). 0.96 (3H. s, H-25), 0.99 (3H, s. H-23); EIMS $m \mathrm{z}$ (\%): $384[\mathrm{M}]^{-}(17) .369(7), 206(5) .191$ (100), $177(9) .149$ (18). $123(22) .95(25), 81(24)$.

$\mathbf{5} \beta$-24S-Ethylchlestan-3 $\beta$-ol (6). Colorless needles $\left(\mathrm{CHCl}_{3}\right)$, ${ }^{3} \mathrm{H}-\mathrm{NMR}(400 \mathrm{MHz}, \mathrm{CDCl} 3) \delta 4.10(\mathrm{lH} . \mathrm{C}-3), 0.64$ (3H. s, H-18). 0.96 (3H. s, H-19), $0.90(3 \mathrm{H}$, d. $J=6.5 \mathrm{~Hz}, \mathrm{H}-2 \mathrm{l})$, $0.82(3 \mathrm{H}$, d. $J=7.7 \mathrm{~Hz}, \mathrm{H}-26), 0.80(3 \mathrm{H} . \mathrm{d}, J=7.7 \mathrm{~Hz}$. H$27) .0 .85(3 \mathrm{H} . \mathrm{t} . J=7.5 \mathrm{~Hz}, \mathrm{H}-29)$ : EIMS $m z(\%): 4 \mathrm{l} 6[\mathrm{M}]^{-}$ (32). 402 (13), 401 (36), 383 (22). $316(7), 290(7), 248(8)$, $233(71), 215(100), 165(19), 107(67) .81(64), 69(44)$.

24 $\alpha$-Ethylchlestan-3 $\alpha-0$ l (7). Colorless needles $\left(\mathrm{CHCl}_{3}\right)$, ${ }^{1} \mathrm{H}$ NMR (400 MHz. $\left.\mathrm{CDCl}_{3}\right) \delta 0.89(3 \mathrm{H}$. d. $J=6.6 \mathrm{~Hz}, \mathrm{H}-$ $21) .0 .84(3 \mathrm{H}, \mathrm{t}, J=7.6 \mathrm{~Hz} . \mathrm{H}-29) .0 .82(3 \mathrm{H}, \mathrm{d}, J=7.6 \mathrm{~Hz}$, $\mathrm{H}-26) .0 .80$ (3H. d. $J=6.8 \mathrm{~Hz}, \mathrm{H}-27), 0.75$ (3H. s, H-19). 0.63 (3H. s. H-18): EIMS $m z(\%): 416[\mathrm{M}]^{+}(100) .401(50)$, 383 (44). $316(8), 290$ (18). 248 (25). 233 (64), 215 (57), 165 (45). $107(47) .83(26), 69(33)$.

Abietan-18 $\beta$-oic acid (8). Colorless needles $\left(\mathrm{CHCl}_{3}\right) .{ }^{1} \mathrm{H}-$ NMR (400 MHz. CDCl $) \delta 0.83$ (6H. d. $J=7.0 \mathrm{~Hz}, \mathrm{H}-16$, 17). 0.84 (3H, s. H-20). 1.17 (3H, s. H-19); EIMS $m z(\%)$ : $306 \mathrm{CM}^{-}(5), 191(\mathrm{l} 6), 163(100), 164$ (16). $149(6) .121$ (11). 109 (13). 95 (16). 81 (18).

2-Allyl-4,5-(methylenedioxy)phenol (10). Yellow oil ${ }^{1} \mathrm{H}-\mathrm{NMR}\left(400 \mathrm{MHz}, \mathrm{CDCl}_{2}\right) \delta 6.5 \mathrm{l}(\mathrm{lH}, \mathrm{s} . \mathrm{H}-3), 6.35(\mathrm{lH}$, s. H-6), $5.77(2 \mathrm{H}, \mathrm{s}, \mathrm{H}-7), 3.22\left(2 \mathrm{H}, \mathrm{d}, J=4.84 \mathrm{~Hz}, \mathrm{H}-\mathrm{l}^{\prime}\right)$ $5.88\left(1 \mathrm{H}, \mathrm{m} . \mathrm{H}-2^{\prime}\right), 5.00(1 \mathrm{H}$, br s. H-3'). 4.97 (1H. m. H-3'): EIMS $m z(\%): 178[\mathrm{M}]^{+}(14), 177(22), 164(26) .163(28)$, $161(7) .151(14) .138(7)$

6-Alyl-6-(3-methylbut-2-enyl)benzo[d] $[1,3]$ dioxol-5(6H)one (11). Colorless oil, $[\alpha]_{\mathrm{D}}^{-1-5}+54\left(\mathrm{c} 0.37, \mathrm{CHCl}_{3}\right)$ : ${ }^{1} \mathrm{H}$ NMR (400 MHz, $\left.\mathrm{CDCl}_{3}\right) \delta 5.45$ (lH. s. H-3). 5.64 (lH. s, H6). 2.63 (lH, dd. $J=7.2 .13 .2 \mathrm{~Hz}, \mathrm{H}-7$ ). 2.28 (lH. dd, $J=$ $7.2 .13 .2 \mathrm{~Hz} . \mathrm{H}-7), 5.57$ (1H. m. H-8). 5.05 (1H. br s. H-9). $4.99(1 \mathrm{H}, \mathrm{m}, \mathrm{H}-9), 2.54$ (1H. dd. $J=7.4,13.7 \mathrm{~Hz}, \mathrm{H}-10)$. $2.23(1 \mathrm{H}$. dd. $J=7.4 .13 .7 \mathrm{~Hz}, \mathrm{H}-10) .4 .94(1 \mathrm{H}, \mathrm{t} . J=7.4 \mathrm{~Hz}$, H-11). 1.60 (3H. s. H-13). $1.66(3 \mathrm{H}, \mathrm{s} . \mathrm{H}-14) .5 .84$ (2H. d. $J$ $=4.5 \mathrm{~Hz}, \mathrm{H}-15)$ : EIMS $m z(\%): 246[\mathrm{M}]^{-}(5) .205(6) .189$ (6). 179 (17). $178(100)$. 177 (30). 151 (12). $147(21) .69$ (23).

Bis(2-ethylhexyl) phthalate (12). Colorless oil. ' $\mathrm{H}$ NMR $\left(\mathrm{l} 00 \mathrm{MHz}, \mathrm{CDCl}_{3}\right) \delta 7.69(2 \mathrm{H}$, dd. $J=3.4 .5 .7 \mathrm{~Hz}, \mathrm{H}-2)$, $7.52(2 \mathrm{H} . \mathrm{dd}, J=3.4,5.7 \mathrm{~Hz} . \mathrm{H}-3) .4 .20(4 \mathrm{H}, \mathrm{d}, J=6.5 \mathrm{~Hz}$, H-1'), $1.66\left(2 \mathrm{H}, \mathrm{m}, \mathrm{H}-2^{\prime}\right), 1.28-1.38\left(16 \mathrm{H}, \mathrm{m}, \mathrm{H}-3^{\prime}, 4^{\prime}, 5^{\prime}, 7^{\prime}\right)$. 
$0.91\left(6 \mathrm{H}, \mathrm{t} . J=7.4 \mathrm{~Hz}, \mathrm{H}-8^{\prime}\right) .0 .88$ (6H. t. $\left.J=7.8 \mathrm{~Hz} . \mathrm{H}-6^{\prime}\right)$ : EIMS $m z(\%): 390\left[\mathrm{M}^{-}\right.$(2), $279(10) .167(35) .149(100)$. $113(10), 83(6), 57(16)$.

Acknowledgments. This research was supported by the National Natural Science Foundation of China (No. 40403014). We thank the analytical group of the State Key Laboratory of Phytochemistry and Plant Resources in West China. Kunming Institute of Botany. Chinese Academy of Sciences for all spectra tests.

\section{References}

1. Otto, A.: White. J. D.: Simoneit B. R. T. Science 2002, 297, 1543.

2. Otto, A.: Simoneit. B. R. T. Geodhimica et Gosmochimica Acta 2001, 65,3505

3. Giannasi. D. E.: Niklas. K. J. Science 1977. 197.765.

4. Niklas. K. J.: Giannasi. D. E. Science 1977. 196.877.

5. Niklas. K. J.: Giannasi. D. E. Amer. J. Bot. 1978. 65.943.

6. Zhao, Y. X.: Li. C. S: Luo, X. D.: Wang, Y. F: Zhoul. J. Journal of hitegrative Plant Biology $2006,48,983$

7. Zhao, Y. X.: Li. C. S.: Luo. X. D.: Zhoul. J. Heteroncles 2004. 63. 861

8. Zhao. Y. X.: Li. C. S.: Luo. X. D.: Y1. T. M.: Zhou. J. Hetretica Chimica tcta 2005, 88,325.

9. Wu. Z. Y.: Yin, W. Q: Bao. S. Y.: Tao, D. D.: Yuan, S. H.: Deng.
X. F.: Yuan1. S. X.: You. H. Z.: Lin1. Q. Index Flonoe Immutentsis (Section 1): Yunnan People's Press: 1984: $\mathrm{p} 7$.

10. Hanke. F. J.: Kubo. I. J. Nat. Prod. 1989. 52.1237

11. Chang. J: Xuan. L. J.: Xu, Y. M: Zhang. J. S. Planta Med. 2002. 68.425

12. Sy. L. K.: Brown. G. D. Phyochemistry 1999. 50, 781.

13. Shicjima. K.: Suzuki. M.: Matsumura. T:: Ageta. H. Chem. Phorm. Bull. 1994. 42.377.

14. Seidel. S. B.: Proudfoot. J. R.: Djerassi. C.: Sica. D.: Sodano. G. Stemoids 1986, 77,49 .

15. Yano. K.; Akihisa. T; Kawaguehi. R.; Tamura. T:; Matsumoto. T. Phytochemisty 1992,31, 1741

16. Lu. T.: Vargas. D.: Franzblau. S. G.: Fischer. N. H. Phochemistry 1995. 38.451

17. Seo. S.: Uomori. A.: Iwatani. K: Nakagawa. Y: Takeda. K.: Sankawa U. Plytochemistry 1992.31.3029.

18. Iver. M. R.: Trivedi, G. K. Bull. Chem. Soc. Jpn. 1992, 65. 1662

19. Yakushijin, K; Tohshima. T; Kitagawa. E.: Suzuki, R; Selikawa. J: Morishita. T: Murata. T.: Lu. S. T.: Furukawa. H. Chent. Pharnt Bull. 1984.32.11.

20. Boxuidothoudt. B. C. B. J. Chem. Soc. Perk. I 1980. 2179

21. Albro. P. W: Jordan. S.: Corbett, J. T; Schroeder. J. L. Anal. Chem. 1984, 56, 247

22. Nazir. D. J.; Alearaz, A. P.: Bierl. B. A.; Beroza, M.; Nair. P. P. Biochentistry 1971. 10.4228.

23. Tomita. I.: Nakamura. Y.: Yagi. Y. Ecotoricol. Emin Saf. 1977. 1. 275

24. Kim. S. W: Petersen. R. V.: Lee. E. S. J. Pham. Sci. 1976, 670. 